\title{
The Effect of Mesenchymal Stem Cells Derived Microvesicles on the Treatment of Experimental CCL4 Induced Liver Fibrosis in Rats
}

\author{
Dina Sabry ${ }^{1}$, Abbas Mohamed ${ }^{1}$, Manar Monir ${ }^{1}$, Heba A. Ibrahim ${ }^{2}$ \\ ${ }^{I}$ Department of Medical Biochemistry and Molecular Biology, Faculty of Medicine, Cairo University, Giza, Egypt \\ ${ }^{2}$ Department of Pathology, Faculty of Medicine, Cairo University, Giza, Egypt
}

Background and Objectives: The release of microvesicles (MVs) from mesenchymal stem cells (MSCs) has been implicated in intercellular communication, and may contribute to beneficial paracrine effects of stem cell-based therapies. We investigated the effect of administration of MSC-MVs on the therapeutic potential of carbon tetrachloride ( $\left.\mathrm{CCL}_{4}\right)$ induced liver fibrosis in rats.

Methods: Our work included: isolation and further identification of bone marrow MSC-MVs by transmission electron microscopy (TEM). Liver fibrosis was induced in rats by $\mathrm{CCl}_{4}$ followed by injection of prepared MSC-MVs in injured rats. The effects of MSC-MVs were evaluated by biochemical analysis of liver functions, RNA gene expression quantitation for collagen-1 $\alpha$, transforming growth factor $\beta$ (TGF- $\beta$ ), interleukin-1 $\beta$ (IL-1 $\beta$ ), vascular endothelial growth factor (VEGF) by real time reverse transcription PCR (RT-PCR) techniques. Finally histopathological examination of the liver tissues was assessed for all studied groups.

Results: BM-MSC-MVs treated group showed significant increase in serum albumin levels, VEGF quantitative gene expression $(\mathrm{p}<0.05)$, while it showed a significant decrease in serum alanine transaminase (ALT) enzyme levels, quantitative gene expression of TGF- $\beta$, collagen- $\alpha$, IL-1 $\beta$ compared to CCL 4 fibrotic group $(\mathrm{p}<0.05)$. Additionally, the histopathological assessment of the liver tissues of BM-MSC-MVs treated group showed marked decrease in the collagen deposition \& improvement of histopathological picture in comparison with $\mathrm{CCL}_{4}$ fibrotic group.

Conclusions: Our study demonstrates that BM-MSC-MVs possess anti-fibrotic, anti-inflammatory, and pro-angiogenic properties which can promote the resolution of $\mathrm{CCL}_{4}$ induced liver fibrosis in rats.

Keywords: Mesenchymal stem cells, Microvesicles, Liver fibrosis

Received: December 24, 2018, Revised: June 22, 2019,

Accepted: July 1, 2019, Published online: August 31, 2019

Correspondence to Dina Sabry

Department of Medical Biochemistry and Molecular Biology,

Faculty of Medicine, Cairo University, 1 Gamaa Street, Giza 12613, Egypt

Tel: +21111200200, Fax: +236322697

E-mail: dinasabry@kasralainy.edu.eg

(c) This is an open-access article distributed under the terms of the Creative Commons Attribution Non-Commercial License (http://creativecommons.org/ licenses/by-nc/4.0/), which permits unrestricted non-commercial use, distribution, and reproduction in any medium, provided the original work is properly cited.

Copyright (c) 2019 by the Korean Society for Stem Cell Research

\section{Introduction}

Liver fibrosis results from many chronic injuries and frequently progresses to cirrhosis, liver failure, portal hypertension, and hepatocellular carcinoma. Liver transplantation is the only beneficial treatment for patients with advanced stages of liver fibrosis (1), but this treatment is associated with many obstacles, such as donor shortage, surgical complications, immunological rejection and high medical expenses (2). Thus, finding new therapeutic approaches for liver fibrosis is essential. The use of cell therapy is becoming more prevalent (3). For in- 
stance, hepatocyte transplantation can be used to reinstate liver functions because of the regeneration abilities of these cells. However, the efficacy of this treatment is limited because hepatocytes easily lose their viability and function when they are cultured in vitro or when they are preserved cryogenically (2). Thus, other types of cells have been investigated in an effort to find an ideal treatment for liver diseases. Researches have shown that stem cell transplantation is an effective treatment for liver fibrosis $(4,5)$. Among the different types of stem cells, mesenchymal stem cells (MSCs) in particular have noticeable advantages in regenerative repair because of their high potential for multipotent differentiation, capacity for self-renewal, and low immunogenicity (6). Over the past few years, an increasing number of studies have assessed the anti-fibrotic potential of MSCs. In vivo studies have emphasized the capability of MSCs to reduce liver fibrosis in animal models $(7,8)$. In vitro studies have been aimed to clarify the underlying mechanisms by which MSCs could control hepatic stellate cells (HSCs) activation (9). Finally, clinical trials have evaluated the efficacy of MSC transplantation for the treatment of liver fibrosis in humans $(8,10)$. Moreover, studies have shown that MSCs do not need to engraft in order to induce recovery. Later, it was revealed that MSCs have vast secretion machineries for anti-inflammatory cytokines, chemokines, growth factors (GFs), and shed microvesicles (MVs) that regulate the immune response (11). Several investigations revealed that MVs from MSCs simulate their immunoregulatory and regenerative action $(12,13)$. Cell-derived MVs are wellknown as components of the cell-to-cell communication network. MVs are small $(0.1 \sim 1 \mu \mathrm{m})$ vesicles shed from the cell membrane by nearly all cell types (14). MVs mediate intercellular communication through paracrine/endocrine mechanisms (15). They work as a vehicle to transfer protein, messenger ribonucleic acid (mRNA), and micro RNA (miRNA) to distant cells, changing the gene expression, proliferation, and differentiation of the recipient cells (16). Moreover, MSC-MVs lack Major Histocompatibility Complex (MHC) class I and II, which allows their allogenic transfusion (17). Upon administration with a therapeutic regimen, MVs mimic the effect of MSCs in different experimental models by inhibiting apoptosis and stimulating cell proliferation. Therefore, it opens novel perspectives in utilizing these MVs in tissue regeneration and repair. In addition, the use of MVs instead of stem cells may represent a safe and possibly more advantageous alternative to cell-therapy approaches (18). The aim of the present work is to assess the role of MSCs-MVs as a new therapeutic agent for tissue repair and functional restora- tion in liver fibrosis.

\section{Materials and Methods}

\section{Preparation, isolation and identification of BM-MSCs in culture}

BM cells were flushed from tibia of white albino female rats (Cuxl: HEL1) of matched age (6 weeks) and weight (150 200 g) with Phosphate-Buffered Saline (PBS). $15 \mathrm{ml}$ of the flushed bone marrow cells was carefully layered on $15 \mathrm{ml}$ Ficoll-Paque (Gibco-Invitrogen, Grand Island, NY), then they were centrifuged for $35 \mathrm{~min}$. at $400 \times \mathrm{g} 5^{\circ} \mathrm{C}$. The upper layer was aspirated leaving the mono nuclear cell (MNC) layer undisturbed at the interphase. The MNC layer was carefully aspirated and washed twice in PBS containing $2 \mathrm{mM}$ ethylene diamine tetra acetic acid (EDTA) and centrifuged for 10 minutes at $200 \times \mathrm{g} 5^{\circ} \mathrm{C}$, then isolated BM-MSCs were cultured and propagated on $25 \mathrm{ml}$ culture flasks in Roswell Park Memorial Institute (RPMI)-1640 medium supplemented with 10\% Fetal Bovine Serum (FBS), $0.5 \%$ penicillin, streptomycin and incubated at $37^{\circ} \mathrm{C}$ and $5 \% \mathrm{CO}_{2}$ until reaching 80 90\% confluence within 7 days (19). Cultured MSCs were characterized by morphology and Fluorescent Activated Cell Sorting (FACS) by assessment positivity of cluster of differentiation $\mathrm{CD}{ }^{+}, \mathrm{CD} 73^{+}, \mathrm{CD} 29^{+}, \mathrm{CD} 105^{+}$and negativity of $\mathrm{CD}_{4} 5^{-}$and $\mathrm{CD} 34^{-}$specific to MSCs.

Additionally, the chondrocyte differentiation was achieved by adipocytes StemPro ${ }^{\circledR}$ chondrogenesis differentiation kit (Gibco, Life Technology, Carlsbad, CA, USA) and they were stained by Alcian blue stain (SigmaAldrich, St Louis, MO, USA). The osteoblasts differentiation was constructed by osteoblast StemPro ${ }^{\circledR}$ osteogenesis differentiation kit (Gibco, Life Technology) and they were stained by Alizarin Red S stain (Sigma-Aldrich).

\section{Preparation and identification of MVs derived from BM-MSCs}

Briefly, MVs were obtained from supernatants of MSCs cultured overnight in RPMI deprived of FBS. To obtain MVs, after centrifugation at $10,000 \times \mathrm{g} 4^{\circ} \mathrm{C}$ for 20 minutes to remove debris, cell-free supernatants were centrifuged at $100,000 \times \mathrm{g} 4^{\circ} \mathrm{C}$ (Beckman Coulter Optima L-90K ultracentrifuge) for one hour at $4^{\circ} \mathrm{C}$, washed in serum-free medium 199 containing N-2-Hydroxy Ethyl Piperazine-N'2-Ethane Sulfonic acid (HEPES) $25 \mathrm{mM}$ (Sigma) and submitted to a second ultracentrifugation in the same conditions (13). MVs were characterized by transmission electron microscopy (TEM) and by assessment of CD63, 
CD81 and CD83 using western blot.

\section{TEM characterization for MVs}

MVs were fixed with $2.5 \%$ glutaraldehyde for $2 \mathrm{~h}$, after being washed; MVs were ultra-centrifuged and suspended in $100 \mu \mathrm{L}$ human serum albumin (HSA). A total of 20 $\mu \mathrm{L}$ of MVs was loaded onto a formvar/carbon-coated grid, negatively stained with $3 \%$ aqueous phosphor-tungstic acid for one minute and observed by TEM (HITACHI, H-7650, Japan),which showed their spheroid morphology and confirmed their size (20).

\section{Western blot characterization of MVs}

The antibody used was antigen affinity-purified polyclonal sheep IgG anti-rabbit CD63, CD81 and CD83 (Catalog no. 0349509; BioLegend, San Diego, California, USA). Protein was extracted from isolated MVs using radioimmunoprecipitation buffer's composition. Twenty nanograms of protein were loaded and separated by sodium dodecyl sulfate-polyacrylamide gel electrophoresis on $4 \sim 20 \%$ polyacrylamide gradient gels. Following incubation in 5\% nonfat dry milk, Tris hydrochloride, $0.1 \%$ Tween 20 for $1 \mathrm{~h}$, primary antibodies (1:500 dilution factor for all target proteins) were added to one of the membranes including specimen samples and incubated at $4^{\circ} \mathrm{C}$ overnight. HRP-conjugated secondary antibody (Goat anti-rabbit IgG- HRP-1mg Goat mab -Novus Biologicals) solution was incubated against the blotted target protein for for $2 \mathrm{~h}$ at room temperature. After being washed six times in $1 \mathrm{X}$ TBS-T, densitometric analysis of the immunoblots was performed to quantify the amounts of CD63, CD81 and CD83 against housekeeping protein $\beta$-actin by image analysis software on the ChemiDoc MP imaging system (version 3) produced by Bio-Rad.

\section{Animals}

Forty five female white albino rats (Cuxl: HEL1) of matched age (6 weeks) and weight $(150 \sim 200 \mathrm{~g})$ were bred and maintained in an air-conditioned animal house with specific pathogen-free conditions, and were subjected to a $12: 12$-h daylight/darkness and allowed unlimited access to chow and water this was conducted after the approval of our local ethical comittee: Institutional Animal Care and Use Committee (IACUC). On day 0, rats were divided into the following groups:

Normal control group: 15 rats received $0.2 \mathrm{ml} / 100 \mathrm{~g}$ body weight of castor oil twice a week for 6 weeks. Then they received $1 \mathrm{ml}$ of PBS twice weekly for 4 weeks.

$\mathrm{CCl}_{4}$ fibrotic group: 15 rats received a dose of 0.2 $\mathrm{ml} / 100 \mathrm{~g}$ body weight of $\mathrm{CCl}_{4}$ (Sigma, St Louis, USA) dissolved in equal volume of castor oil (Sigma, St. Louis, USA) subcutaneously twice a week for 6 weeks (21). Then, they received $1 \mathrm{ml}$ of PBS twice weekly for 4 weeks. Liver fibrosis was determined by histopathological examination.

$\mathrm{CCl}_{4} / \mathrm{BM}-\mathrm{MSC}-\mathrm{MV}$ s treated group: 15 rats received $0.2 \mathrm{ml} / 100 \mathrm{~g}$ body weight of $\mathrm{CCl}_{4}$ by the schedule mentioned above. Then, they were injected by MSC-MVs with a dose of $(4 \mu \mathrm{g} / \mathrm{ml}$ PBS$)$ per rat IV via tail vein twice weekly for 4 weeks (22). The protein content of MVs was quantified by Bradford method (BioRad, Hercules, CA, USA).

After 4 weeks from stopping of $\mathrm{CCl}_{4}$ and administration of MVs, venous blood was collected from the retro-orbital vein. All rats were sacrificed with decapitation, and liver tissues were harvested for analysis.

\section{Biochemical analysis of liver functions}

Alanine Transaminase (ALT) and albumin were assessed according to manual instructions. The ALT kit was provided by (BioMeD, Hannover, Germany, CAT NO: GPT11240), while albumin kit was provided by (BioMed, Hannover, Germany, CAT NO: ALB100200) and they were measured in serum by Colorimetric, Endpoint method.

Table 1. Primer sequence for all the studied genes are the following

\begin{tabular}{lll}
\hline Gene symbol & \multicolumn{1}{c}{ Primer sequence from $5^{\prime}$ to $3^{\prime}$} & Gene bank accession number \\
\hline TGF- $\beta$ & F: TGCGCCTGCAGAGATTCAAG & NM021578.2 \\
& R: AGGTAACGCCAGGAATTTTGCTA & NM031512.2 \\
IL-1 $\beta$ & R: GCTGTGGCAGCTACCTATGTCTTG & \\
& F:AGAGCGTCATCATCCCACGAG & KJ696743.1 \\
Collagen-1 $\alpha$ & R:CCTTCTTGAGGTTGCCAGTC & \multirow{2}{*}{ XM011354722.1 } \\
VEGF & R:TCTGGACATCTTCCAGGAGTA & \multirow{2}{*}{ XR598347.1 } \\
FAPDH & R:CACCCTGTTGCTGTAGCCATCATATTC & \\
\hline
\end{tabular}




\section{RNA extraction}

RNA extraction kit was provided from Thermo Fisher Scientific Inc. Germany (GeneJET, Kit, \#K0732). Liver tissue RNA was purified according to manual instructions.

\section{Quantitative real time-PCR (qRT-PCR) assessment}

The kit was provided by Bioline, a median life science company, UK (SensiFAST ${ }^{\text {TM }}$ SYBR $^{\circledR}$ Hi-ROX One-Step Kit, catalog no. PI-50217 V) which is formulated for highly reproducible first-strand cDNA synthesis and subsequent real-time PCR in one step.

RT-PCR master mix was prepared by $10 \mu 12 \mathrm{x}$ SensiFAST $^{\text {TM }}$ SYBR (final concentration was $1 \mathrm{x}$ ) then $0.8 \mu 1$ of $10 \mu \mathrm{M}$ forward Primer, $0.8 \mu 1$ of $10 \mu \mathrm{M}$ reverse Primer (primers sequence was shown in Table 1), $0.2 \mu 1$ of reverse transcriptase, $0.4 \mu 1$ of RiboSafe RNase inhibitor, $3.8 \mu 1$ of $\mathrm{H}_{2} \mathrm{O}$ and $4 \mu \mathrm{l}$ of RNA template the final volume was $20 \mu 1$. Finally the prepared reaction mix samples were applied in real time PCR (StepOne Applied Biosystem, Foster city, USA). RT-PCR thermal cycling profile was one cycle for reverse transcription temperature of $45^{\circ} \mathrm{C}$ for 10 minutes, one cycle for polymerase activation at $95^{\circ} \mathrm{C}$ for 2 minutes and finally 40 cycles including (denaturation at $95^{\circ} \mathrm{C}$ for 5 seconds, annealing at $60^{\circ} \mathrm{C}$ for 10 seconds and extension acquired at end of step at $72^{\circ} \mathrm{C}$ for 5 seconds). The RQ of each target gene is quantified according to the calculation of $\Delta \Delta \mathrm{Ct}$. We calculated the $\mathrm{RQ}$ of each gene by $2^{-\Delta \Delta \mathrm{Ct}}$ normalization to house keeping gene.

\section{Analysis of liver histopathology}

Liver samples were collected into PBS and fixed overnight in $40 \mathrm{~g} / \mathrm{L}$ paraformaldehyde in PBS at $4^{\circ} \mathrm{C}$. Serial $5-\mu \mathrm{m}$ sections of the right lobes of the livers were stained with Hematoxylin and Eosin (H\&E) and Sirius red staining and were examined histopathologically at the pathology department.

\section{Statistical analysis}

Data were coded and entered using the statistical package SPSS version 24. Data were summarized using mean and standard deviation for quantitative variables. Comparisons between groups were done using analysis of variance (ANOVA) with multiple comparisons post hoc test (23). Correlations between quantitative variables were done using Pearson correlation coefficient (24). p-values less than 0.05 were considered as statistically significant.

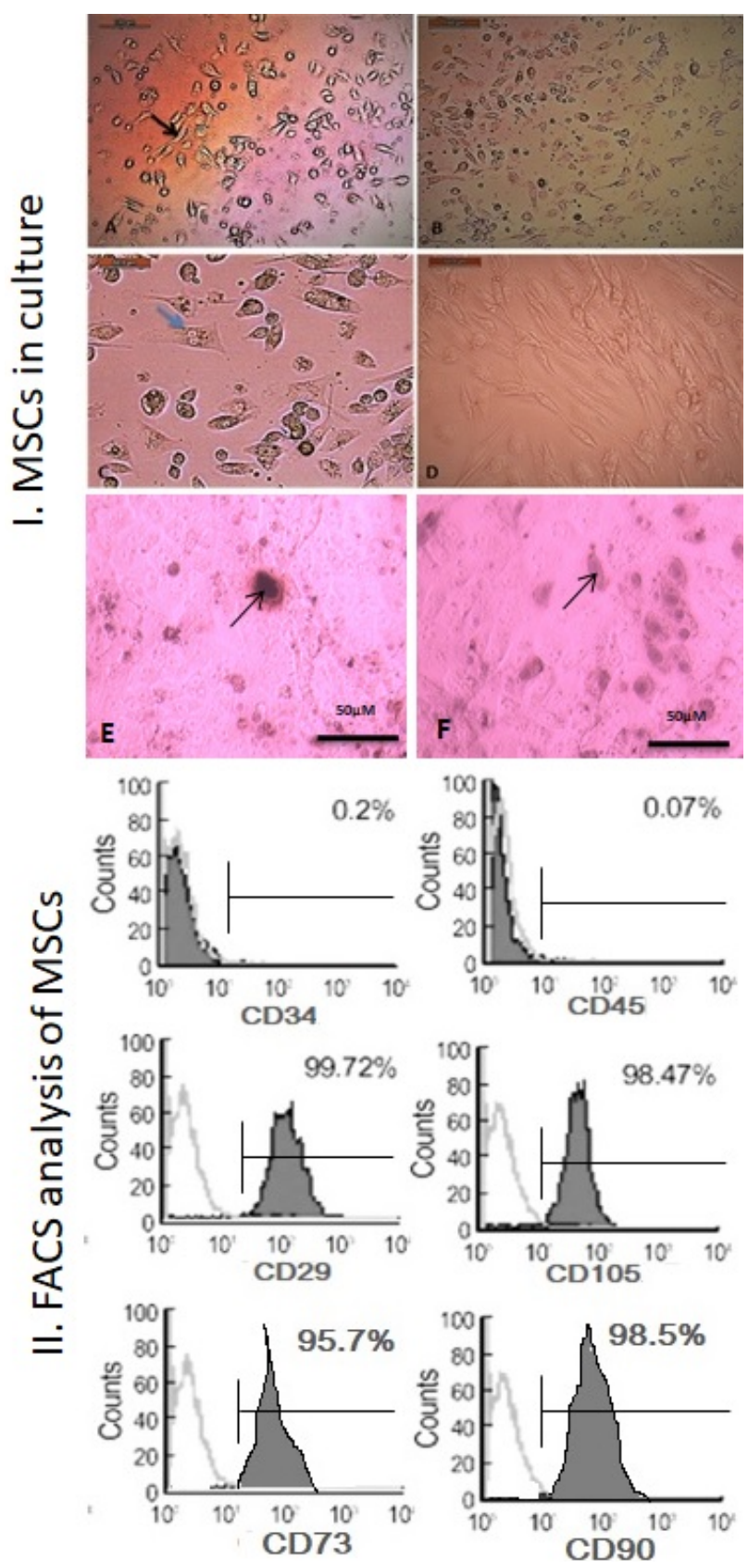

Fig. 1. BM-MSCs in culture. (A) After 24 hour with black arrow to identify MSCs as fibroblast like cell $(100 \times)$, (B) after 72 hour $(100 \times)$, (C) blue arrow to show MSCs during their cell division $(400 \times)$ and (D) after 14 days to show $80 \%$ confluence proliferating MSCs. FACS analysis for cultured MSCs. They were negative for $\mathrm{CD}^{-} 4^{-}(0.2 \%), \mathrm{CD}^{-} 5^{-}(0.07 \%)$ and positive for $\mathrm{CD} 29^{+}$ (99.72\%), $\mathrm{CD} 105^{+}(98.4 \%), \mathrm{CD}^{+}{ }^{+}(95.7 \%)$ and $\mathrm{CD}^{+}(98.5 \%)$. (E) MSCs were differentiated into osteoblasts stained with Alizarin Red S stain (black arrow) $(100 \times)$ ). (F) MSCs were differentiated into chondrocytes stained with Alcian blue stain (black arrow) $(100 \times)$. 


\section{Results}

\section{BM-MSCs isolation, propagation and identification}

MSCs were isolated, propagated, and identified by their morphology as fibroblast spindle shape cells and colony forming units (Fig. 1 (I): A D). MSCs were differentiated into osteoblasts stained with Alizarin Red S stain and chondrocytes stained with Alcian blue (Fig. 1 (I): E, F respectively). Furthermore phenotypic FACS analysis showed that MSCs were uniformly positive for specific MSCs surface marker CD29, CD105, CD73, CD90 and negative for CD34, CD45 (hematopoietic marker) (Fig. 1 (II)).

\section{Identification of isolated BM-MSCs-MVs}

$\mathrm{BM}-\mathrm{MSCs} / \mathrm{MVs}$ were characterized by their expression of specific markers CD63, CD81 and CD83 as detected by western blot (Fig. 2A). Furthermore MVs were identified using TEM by their homogenous size $(500 \mathrm{~nm})$ and their spheroid morphology (Fig. 2B).

\section{Biochemical analysis of liver functions}

Serum albumin level showed a significant decrease in $\mathrm{CCL}_{4}$ fibrotic group compared to the normal control group $(p<0.05)$. However, there was a significant increase in albumin level after 1 month of BM-MSC-MVs administration in the fibrotic rats compared to $\mathrm{CCL}_{4}$ fibrotic group $(\mathrm{p}<0.05)$, and it was non-statistically significant compared to the normal control group ( $\mathrm{p}>0.05)$ (Fig. 3A). ALT serum levels showed a significant increase in the $\mathrm{CCL}_{4}$ fibrotic group compared to the normal control group $(p<0.05)$. However, after 1 month of administration of BM-MSC-MVs in the fibrotic rats showed a significant decrease compared to the $\mathrm{CCL}_{4}$ fibrotic group $(\mathrm{p}<0.05)$, but, it was still significantly higher than the normal control group $(\mathrm{p}<0.05)$ (Fig. 3B).

\section{Real time PCR}

Quantitative gene expression of fibrotic markers TGF- $\beta$ and collagen- $1 \alpha$ in rats' livers showed significant increase in $\mathrm{CCL}_{4}$ fibrotic group in comparison with the normal control group $(p<0.05)$. Conversely, they showed significant decrease after 1 month of administration of BM-MSC-MVs in the fibrotic rats compared to the $\mathrm{CCL}_{4}$ fibrotic group $(p<0.05)$. However, the gene expression of TGF- $\beta$ was still significantly higher than the normal control group $(\mathrm{p}<0.05)$ (Fig. 4A, 4B). As regard VEGF gene expression showed a significant decrease in $\mathrm{CCL}_{4}$ fibrotic group compared to the normal control group $(\mathrm{p}<0.05)$. On the other hand, it showed a significant increase after

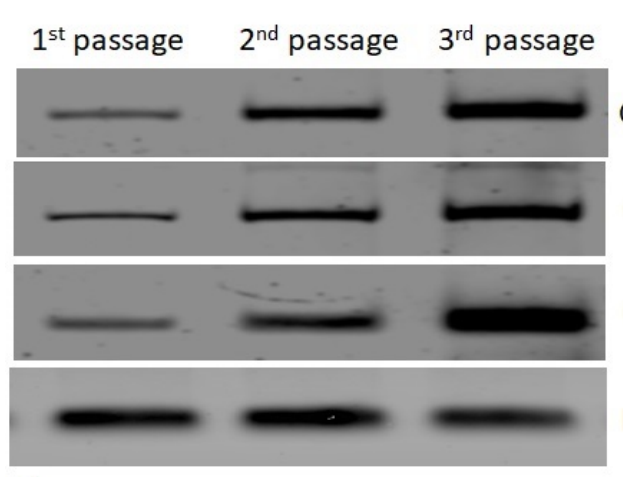

A

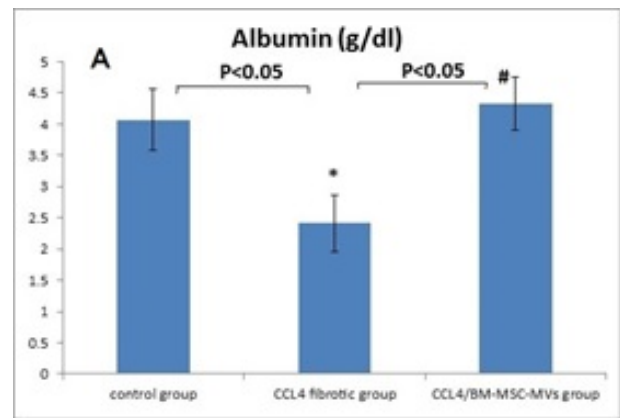

CD63

CD81

CD83

B-actin

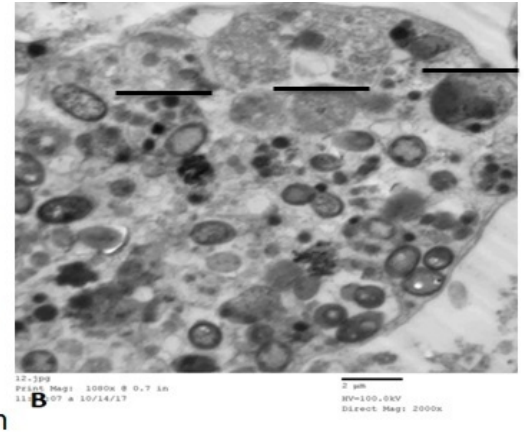

Fig. 2. (A) Western blots scanning densitometry for CD61, CD80 and CD83 for 3 different passages of MVs derived BM-MSCs. (B) TEM of $M V_{s}$; they were spheroid (- 500 $\mathrm{nm}$ ) and have well defined membrane with heterogeneous contents.

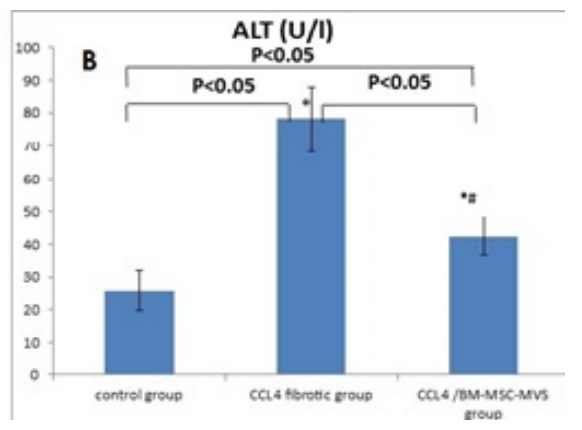

Fig. 3. (A) Serum Albumin levels (g/dl) in all studied groups. (B) Serum ALT levels (U/l) in all studied groups. 

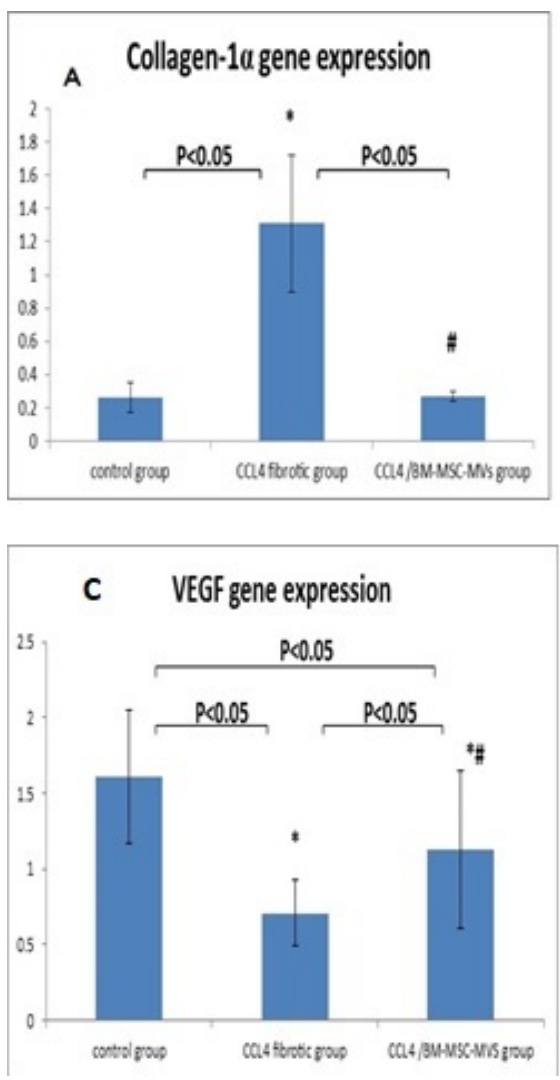
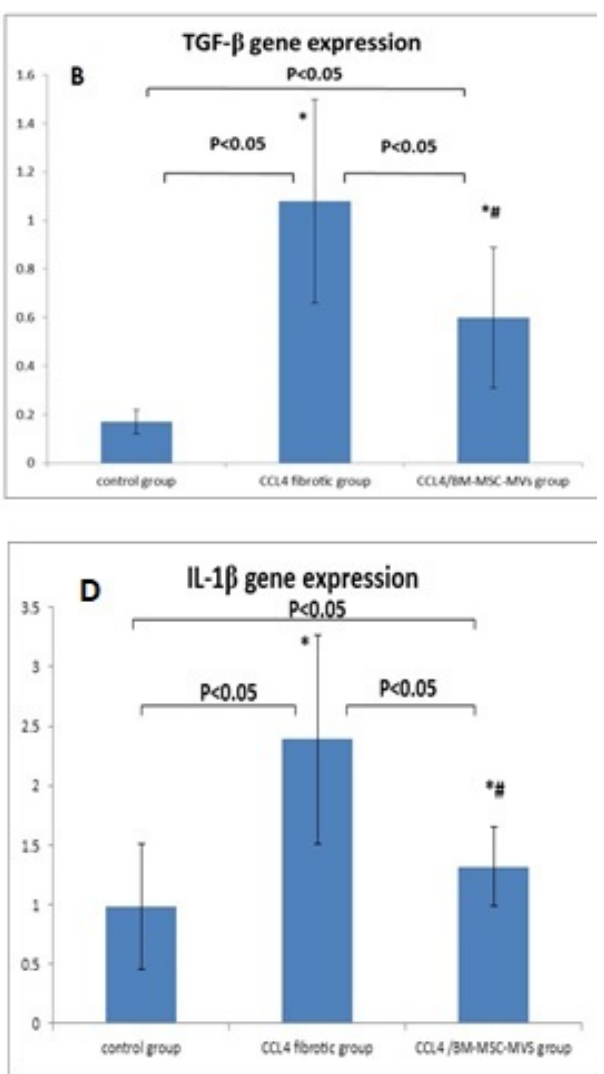

Fig. 4. (A) qRT-PCR genes expression of collagen- $1 \alpha$, (B) TGF- $\beta$, (C) VEGF and (D) IL-1 $\beta$ in liver tissues of all studied groups.
1 month of the administration of BM-MSC-MVs in the fibrotic rats compared to the $\mathrm{CCL}_{4}$ fibrotic group $(\mathrm{p}<0.05)$, however, it was significantly lower than its expression in the normal control group $(\mathrm{p}<0.05)$ (Fig. 4C). Concerning the gene expression of IL- $\beta$ in rats' livers, it showed a significant increase in $\mathrm{CCL}_{4}$ fibrotic group compared to the normal control group $(p<0.05)$. In contrast, it showed a significant decrease after 1 month of administration of BM-MSC-MVs in the fibrotic rats in comparison with the $\mathrm{CCL}_{4}$ fibrotic group $(\mathrm{p}<0.05)$, however, it was significantly higher than its expression in the normal control group $(\mathrm{p}<0.05)$ (Fig. 4D).

Finally, the histopathological examination of liver tissues from all studied groups revealed that BM-MSC-MVs have a significant anti-fibrotic effect, demonstrated in H\&E and Sirius red stained sections, and evidenced by remarkable reduction in the size of portal fibrous expansion and regression of septal fibrosis with improvement of liver histopathological picture compared to $\mathrm{CCL}_{4}$ fibrotic group as shown in (Fig. 5).

\section{Discussion}

The application of MSCs therapy in liver fibrosis treat- ment has been increasingly investigated in the recent years. MSCs obtained from a variety of sources (e.g. bone marrow, umbilical cord (UC) blood and adipose tissue) have been studied and have achieved remarkable results (25). However, accumulating evidence indicates that MSCs release extracellular vesicles (EVs) that deliver genes, miRNAs and proteins to recipient cells, acting as mediators of MSCs paracrine actions (26), and thus, may be harnessed for therapeutic purposes in a similar fashion to their parent cells. Furthermore, in comparison to whole cell-based therapies, MSC-EVs may offer specific advantages for patient safety such as lower propensity to trigger innate and adaptive immune responses and inability to directly form tumors (27).

EVs are subcategorized into three groups; exosomes, MVs and apoptotic bodies, each of which have different biological impact (28). MVs derived from MSCs have shown hopeful therapeutic effects in various animal models. For example, lung injury (29), kidney injury (30, 31), and cardiovascular diseases (32). Despite these interesting studies, we have a limited understanding of the effects of MSC-MVs in experimental models of liver fibrosis.

Our present study was conducted to investigate the effect of murine bone marrow (mBM)- MSC-MVs on the 

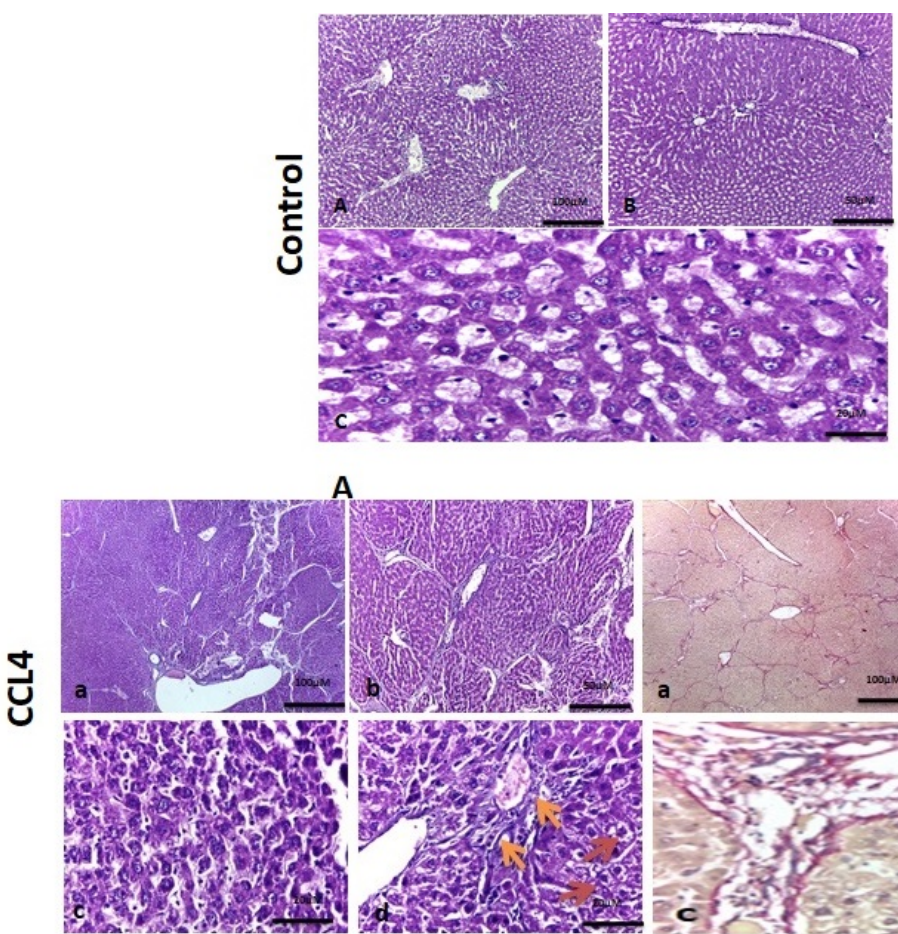

B

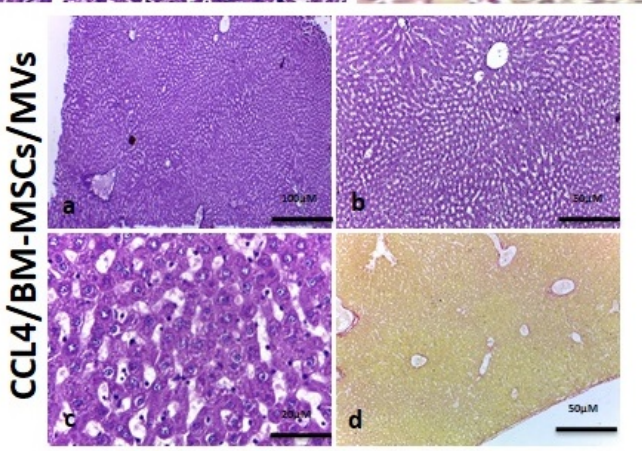

Fig. 5. Divided into 3 panels. First panel: Histopathology of liver tissue in control group stained with H\&E: a $(40 \times), b(100 \times)$ and C $(400 \times)$ showing normal hepatic architecture, normal hepatocytes, portal tracts and central veins. Second panel: (A) Histopathology of liver tissue in $\mathrm{CCL}_{4}$ fibrotic group stained with H\&E: $\mathrm{a}(40 \times)$ and $\mathrm{b}(100 \times)$ showing loss of normal hepatic architecture, marked fibrous septations with complete nodules formation and fibrous expansion of the portal tracts, c $(400 \times)$ showing dysplastic changes of hepatocytes in the form of nuclear hyperchromasia and pleomorphism (green arrows), $d(400 \times)$ showing hydropic degeneration of hepatocytes (red arrows) and periportal lymphocytic infiltration (orange arrows). (B) Histopathology of liver tissue in $\mathrm{CCL}_{4}$ fibrotic group stained with Sirius red: a $(40 \times), b(100 \times)$ showing marked fibrous septations with complete nodules formation, c $(400 \times)$ showing fibrous expansion of the portal tract. Third panel: Histopathology of liver tissue in BM-MSC-MVs treated group stained with H\&E: a $(40 \times), b(100 \times)$ showing preserved liver architecture and no fibrosis, c $(400 \times)$ showing thin plate of normal hepatocytes and no inflammation. Sirius red staining: $d(100 \times)$ showing no fibrosis \& the Sirius red stain is only limited to the outline of blood vessels.

treatment of $\mathrm{CCL}_{4}$ induced liver fibrosis in experimental rats. Thereby, we assessed their potential utility as therapeutic agents for tissue repair and functional restoration in liver fibrosis.

Results revealed that BM-MSC-MVs administration at a dose of (4 $\mu \mathrm{g} / \mathrm{ml}$ PBS via tail vein) recovered liver function, architectural changes and resolved fibrous damage via down regulation of the gene expression of TGF- $\beta$ (fibrosis marker), Collagen-1 $\alpha$ (fibrosis marker), IL-1 $\beta$ (inflammatory marker) and up regulation of the gene expression of VEGF (angiogenesis marker).

In our present study, when rats were treated with $\mathrm{CCL}_{4}$ twice weekly for 6 weeks, the liver exhibited a marked increase in extracellular matrix (ECM) contents and displayed bundles of collagen surrounding the hepatic lobules leading to large fibrous septa as seen by the histopathological examination. Additionally, there was a significant over expression of TGF- $\beta$ and collagen- $1 \alpha$ genes 
in the rats' livers as assessed by qRT-PCR. All of these are characteristics of advanced fibrosis and early cirrhosis. There was also evidence of liver dysfunction, as reflected by the significantly decreased serum albumin levels and the significantly increased serum ALT levels when compared to the normal control group $(\mathrm{p}<0.05)$. These results are in agreement with those of Dong et al. (33) and Shrestha et al. (34) who induced liver fibrosis in rats and mice respectively using $\mathrm{CCL}_{4}$.

Lei et al. (35) characterized the MVs released from BM-MSC by FACS, they were positive for the MSC markers (CD90, CD44 and CD73) and negative for CD34 and CD45 (hematopoietic markers). Moreover, MSC-MVs lack MHC class I and II, which facilitates their allogenic transfusion.

In our study the isolation of BM-MSC-MVs was successful, confirmed by their positivity for CD90 and their negativity for CD45, and their size $(500 \mathrm{~nm})$ as detected by TEM, and they were transplanted into the $\mathrm{CCL}_{4}$ fibrotic rats with no evidence of immune rejection.

Milosavljevic et al. (7) stated that MSCs could improve $\mathrm{CCL}_{4}$ induced liver fibrosis in mice. In that study, Sirius red staining of liver tissues obtained from $\mathrm{CCl}_{4}$-treated group revealed extensive collagen deposition and pseudolobular formation. While, stained areas of fibrous dense tissue were reduced in MSC-treated mice.

Besides, Haga et al. (36) informed that either human MSC-EVs or murine MSC-EVs could achieve anti-inflammatory effects. The histological examination by $\mathrm{H} \& \mathrm{E}$ of D-Galactosamine/ $\alpha$-TNF treated mice livers revealed profound hepatocellular apoptosis, hemorrhagic necrosis, enucleated necrosis, and mononuclear leukocyte infiltration with cytoplasmic vacuolization. In contrast, livers from mice treated with either hMSC-EVs or mMSC-EVs had less inflammation.

Consistent with the previous studies, our histopathological examination by $\mathrm{H} \& \mathrm{E}$ and Sirius red staining of liver tissues of BM-MSC-MVs treated group showed that they have a significant anti fibrotic and anti- inflammatory effects as evidenced by remarkable reduction in the size of portal fibrous expansion and regression of septal fibrosis with improvement of liver histopathological picture compared to $\mathrm{CCL}_{4}$ fibrotic group, and thus, emphasizing that the anti-fibrotic and the anti-inflammatory effects of MSCs may be attributed to their shedding MVs.

The pro inflammatory cytokine IL- $\beta$ is an important participant, along with other cytokines, in controlling the progression from liver injury to fibrogenesis through activation of HSCs in vivo (37). Therefore, targeting IL-1 $\beta$ signaling may be a potentially valuable therapeutic strat- egy in chronic liver diseases (CLDs) (38).

Song et al. (39) reported that MSC transplantation in a partially hepatectomized swine model enhanced liver regeneration in the early acute liver failure stage and showed a significant decrease in IL-1 $\beta$ serum levels, suggesting that MSCs anti-inflammatory actions may mimic its effects in hepatic regeneration.

Added to that, Chen et al. (40) showed that MSC-CM administration significantly reduced the mRNA expression levels of IL- $1 \beta$ in irradiated rats' livers and could prevent further liver injury.

In our study, BM-MSC-MVs treated group showed significant decrease in IL-1 $\beta$ gene expression compared to the $\mathrm{CCL}_{4}$ fibrotic group $(\mathrm{p}<0.05)$. However, it was still significantly higher than the control group $(\mathrm{p}<0.05)$. Taken together, MSCs through their shedding MVs can modulate the inflammatory response by decreasing IL-1 $\beta$ expression so that they can minimize the hepatocytes injury, enhance their survival and decrease the activation of HSCs, which is beneficial in the treatment of liver fibrosis.

The development of liver fibrosis is associated with decreased hepatic VEGF gene expression as well as sinusoidal rarefaction of the fibrotic scar. In contrast, the resolution of fibrosis is characterized by a rise in hepatic VEGF levels and revascularization of the fibrotic tissue.

Additionally, DeLeve (41) reported that VEGF is essential for the maintenance of liver mass during chronic injury through the enhancement of liver regeneration. So that increasing VEGF expression may be helpful in the treatment of chronic liver diseases (CLDs) and fibrosis.

Adas et al. (42) informed that after the injection of MSCs and VEGF-transfected MSCs into the portal vein following liver resection, they were engrafted in the liver and they increased bile duct and liver hepatocyte proliferation, supported liver function and liver volume/ weight via secretion of many GFs including the VEGF.

Moreover, Lee et al. (43) stated that hypoxic CM from human adipose tissue MSCs infusion in partially hepatectomized rats significantly increased the expression of VEGF which enhanced angiogenesis and liver regeneration.

The studies that clarify the role of MSC-MVs in promoting angiogenesis and increasing VEGF expression in liver injury and fibrosis are rare, but there are studies showing their pro-angiogenic effects in other organs (44, 45).

Our study results revealed that the treatment with BM-MSC-MVs significantly increased VEGF gene expression in rats' livers compared to the $\mathrm{CCL}_{4}$ fibrotic 
group $(\mathrm{p}<0.05)$ and accompanied by hepatocytes regeneration \& improvement of the histopathological picture.

Accordingly, we can conclude that MVs are responsible for the angiogenic properties of MSCs via increasing the expression of VEGF and these angiogenic actions can promote hepatocytes regeneration, and decrease liver injury helping to lessen liver fibrosis.

In conclusion, our study clearly demonstrates that BMMSC-MVs possess anti-fibrotic, anti-inflammatory and pro-angiogenic properties which can promote the resolution of $\mathrm{CCL}_{4}$ induced liver fibrosis in rats. Thus, BMMSCs-MVs are new tool for liver fibrosis treatment by a cell free therapy.

This work needs further investigation to clarify the content and the exact mechanism of action of MSC-MVs. Microarray gene analysis has to be done to define which mRNA transcripts are frequently present so that the intended therapeutic effect can be translated and optimized. In vivo tracking of MSC-MVs by fluorescence labeling has to be done. Finally, additional experiments are required to investigate long-term safety of the administration of MSC-MVs.

\section{Potential Conflict of Interest}

The authors have no conflicting financial interest.

\section{References}

1. Koyama Y, Xu J, Liu X, Brenner DA. New developments on the treatment of liver fibrosis. Dig Dis 2016;34:589-956

2. Eom YW, Kim G, Baik SK. Mesenchymal stem cell therapy for cirrhosis: present and future perspectives. World J Gastroenterol 2015;21:10253-10261

3. Guo Y, Chen B, Chen LJ, Zhang CF, Xiang C. Current status and future prospects of mesenchymal stem cell therapy for liver fibrosis. J Zhejiang Univ Sci B 2016;17:831-841

4. Rengasamy M, Singh G, Fakharuzi NA, Siddikuzzaman, Balasubramanian S, Swamynathan P, Thej C, Sasidharan G, Gupta PK, Das AK, Rahman AZA, Fakiruddin KS,Nian LM, Zakaria Z, Majumdar AS. Transplantation of human bone marrow mesenchymal stromal cells reduces liver fibrosis more effectively than Wharton's jelly mesenchymal stromal cells. Stem Cell Res Ther 2017;8:143

5. Kharaziha P, Hellström PM, Noorinayer B, Farzaneh F, Aghajani K, Jafari F, Telkabadi M, Atashi A, Honardoost $M$, Zali MR, Soleimani M. Improvement of liver function in liver cirrhosis patients after autologous mesenchymal stem cell injection: a phase I-II clinical trial. Eur J Gastroenterol Hepatol 2009;21:1199-1205

6. Jang YO, Kim MY, Cho MY, Baik SK, Cho YZ, Kwon SO. Effect of bone marrow-derived mesenchymal stem cells on hepatic fibrosis in a thioacetamide-induced cirrhotic rat model. BMC Gastroenterol 2014;14:198

7. Milosavljevic N, Gazdic M, Simovic Markovic B, Arsenijevic A, Nurkovic J, Dolicanin Z, Jovicic N, Jeftic I, Djonov V, Arsenijevic N, Lukic ML, Volarevic V. Mesenchymal stem cells attenuate liver fibrosis by suppressing Th17 cells - an experimental study. Transpl Int 2018;31: 102-115

8. Berardis S, Dwisthi Sattwika P, Najimi M, Sokal EM. Use of mesenchymal stem cells to treat liver fibrosis: current situation and future prospects. World J Gastroenterol 2015;21:742-758

9. Jang YO, Jun BG, Baik SK, Kim MY, Kwon SO. Inhibition of hepatic stellate cells by bone marrow-derived mesenchymal stem cells in hepatic fibrosis. Clin Mol Hepatol 2015;21:141-149

10. Amin MA, Sabry D, Rashed LA, Aref WM, el-Ghobary MA, Farhan MS, Fouad HA, Youssef YA. Short-term evaluation of autologous transplantation of bone marrow-derived mesenchymal stem cells in patients with cirrhosis: Egyptian study. Clin Transplant 2013;27:607-612

11. Wang N, Li Q, Zhang L, Lin H, Hu J, Li D, Shi S, Cui S, Zhou J, Ji J, Wan J, Cai G, Chen X. Mesenchymal stem cells attenuate peritoneal injury through secretion of TSG-6. PLoS One 2012;7:e43768

12. Baglio SR, Pegtel DM, Baldini N. Mesenchymal stem cell secreted vesicles provide novel opportunities in (stem) cell-free therapy. Front Physiol 2012;3:359

13. Bruno S, Grange C, Deregibus MC, Calogero RA, Saviozzi S, Collino F, Morando L, Busca A, Falda M, Bussolati B, Tetta C, Camussi G. Mesenchymal stem cell-derived microvesicles protect against acute tubular injury. J Am Soc Nephrol 2009;20:1053-1067

14. Biancone L, Bruno S, Deregibus MC, Tetta C, Camussi G. Therapeutic potential of mesenchymal stem cell-derived microvesicles. Nephrol Dial Transplant 2012;27:3037-3042

15. Hulsmans M, Holvoet P. MicroRNA-containing microvesicles regulating inflammation in association with atherosclerotic disease. Cardiovasc Res 2013;100:7-18

16. Sabin K, Kikyo N. Microvesicles as mediators of tissue regeneration. Transl Res 2014;163:286-295

17. Kilpinen L, Impola U, Sankkila L, Ritamo I, Aatonen M, Kilpinen S, Tuimala J, Valmu L, Levijoki J, Finckenberg P, Siljander P, Kankuri E, Mervaala E, Laitinen S. Extracellular membrane vesicles from umbilical cord blood-derived MSC protect against ischemic acute kidney injury, a feature that is lost after inflammatory conditioning. J Extracell Vesicles 2013 doi: 10.3402/jev. v2i0.21927

18. Yin H, Jiang H. [Application prospect of stem cell-derived microvesicles in regeneration of injured tissues]. Sheng Wu Yi Xue Gong Cheng Xue Za Zhi 2015;32:688-692. Chinese

19. Abdel Aziz MT, Atta HM, Mahfouz S, Fouad HH, Roshdy NK, Ahmed HH, Rashed LA, Sabry D, Hassouna AA, Hasan NM. Therapeutic potential of bone marrow-derived mesenchymal stem cells on experimental liver fibrosis. Clin 
Biochem 2007;40:893-899

20. Gatti S, Bruno S, Deregibus MC, Sordi A, Cantaluppi V, Tetta C, Camussi G. Microvesicles derived from human adult mesenchymal stem cells protect against ischaemia-reperfusion-induced acute and chronic kidney injury. Nephrol Dial Transplant 2011;26:1474-1483

21. Zhao DC, Lei JX, Chen R, Yu WH, Zhang XM, Li SN, Xiang P. Bone marrow-derived mesenchymal stem cells protect against experimental liver fibrosis in rats. World J Gastroenterol 2005;11:3431-3440

22. Qu Y, Zhang Q, Cai X, Li F, Ma Z, Xu M, Lu L. Exosomes derived from miR-181-5p-modified adipose-derived mesenchymal stem cells prevent liver fibrosis via autophagy activation. J Cell Mol Med 2017;21:2491-2502

23. Chan YH. Biostatistics 102: quantitative data--parametric \& non-parametric tests. Singapore Med J 2003;44:391-396

24. Chan YH. Biostatistics 104: correlational analysis. Singapore Med J 2003;44:614-619

25. Truong NH, Nguyen NH, Le TV, Vu NB, Huynh N, Nguyen TV, Le HM, Phan NK, Pham PV. Comparison of the treatment efficiency of bone marrow-derived mesenchymal stem cell transplantation via tail and portal veins in $\mathrm{CCl}_{4}$-induced mouse liver fibrosis. Stem Cells Int 2016;2016:5720413

26. Nargesi AA, Lerman LO, Eirin A. Mesenchymal stem cell-derived extracellular vesicles for renal repair. Curr Gene Ther 2017;17:29-42

27. Yu B, Zhang X, Li X. Exosomes derived from mesenchymal stem cells. Int J Mol Sci 2014;15:4142-4157

28. Rad F, Pourfathollah AA, Yari F, Mohammadi S, Kheirandish M. Microvesicles preparation from mesenchymal stem cells. Med J Islam Repub Iran 2016;30:398

29. Monsel A, Zhu YG, Gennai S, Hao Q, Hu S, Rouby JJ, Rosenzwajg M, Matthay MA, Lee JW. Therapeutic effects of human mesenchymal stem cell-derived microvesicles in severe pneumonia in mice. Am J Respir Crit Care Med 2015;192:324-336

30. Zou X, Zhang G, Cheng Z, Yin D, Du T, Ju G, Miao S, Liu G, Lu M, Zhu Y. Microvesicles derived from human Wharton's Jelly mesenchymal stromal cells ameliorate renal ischemia-reperfusion injury in rats by suppressing CX3CL1. Stem Cell Res Ther 2014;5:40

31. Salem M, Helal O, Metwaly H, El Hady A, Ahmed S. Histological and immunohistochemical study of the role of stem cells, conditioned medium and microvesicles in treatment of experimentally induced acute kidney injury in rats. J Med Histol 2017;1:69-83

32. Sharma AK, Lu G, Salmon MD, Gehrau RC, Mas VR, Weiss ML, Ailawadi G, Upchurch GR. Human mesenchymal stem cell-derived microvesicles mitigate aortic smooth muscle cell activation via miR-147 and attenuate aortic aneurysm formation. Circulation 2015;132:A11550
33. Dong $\mathrm{S}$, Chen QL, Song YN, Sun Y, Wei B, Li XY, Hu YY, Liu P, Su SB. Mechanisms of $\mathrm{CCl}_{4}$-induced liver fibrosis with combined transcriptomic and proteomic analysis. J Toxicol Sci 2016;41:561-572

34. Shrestha N, Chand L, Han MK, Lee SO, Kim CY, Jeong YJ. Glutamine inhibits $\mathrm{CCl}_{4}$ induced liver fibrosis in mice and TGF- $\beta 1$ mediated epithelial-mesenchymal transition in mouse hepatocytes. Food Chem Toxicol 2016;93:129-137

35. Lei Q, Liu T, Gao F, Xie H, Sun L, Zhao A, Ren W, Guo H, Zhang L, Wang H, Chen Z, Guo AY, Li Q. Microvesicles as potential biomarkers for the identification of senescence in human mesenchymal stem cells. Theranostics 2017;7:2673-2689

36. Haga H, Yan IK, Takahashi K, Matsuda A, Patel T. Extracellular Vesicles from bone marrow-derived mesenchymal stem cells improve survival from lethal hepatic failure in mice. Stem Cells Transl Med 2017;6:1262-1272

37. Senior JR. Alanine aminotransferase: a clinical and regulatory tool for detecting liver injury-past, present, and future. Clin Pharmacol Ther 2012;92:332-339

38. Kang KS. Abnormality on liver function test. Pediatr Gastroenterol Hepatol Nutr 2013;16:225-232

39. Song YM, Lian CH, Wu CS, Ji AF, Xiang JJ, Wang XY. Effects of bone marrow-derived mesenchymal stem cells transplanted via the portal vein or tail vein on liver injury in rats with liver cirrhosis. Exp Ther Med 2015;9:1292-1298

40. Chen J, Li C, Chen L. The role of microvesicles derived from mesenchymal stem cells in lung diseases. Biomed Res Int 2015;2015:985814

41. DeLeve LD. Liver sinusoidal endothelial cells and liver regeneration. J Clin Invest 2013;123:1861-1866

42. Adas G, Koc B, Adas M, Duruksu G, Subasi C, Kemik O, Kemik A, Sakiz D, Kalayci M, Purisa S, Unal S, Karaoz E. Effects of mesenchymal stem cells and VEGF on liver regeneration following major resection. Langenbecks Arch Surg 2016;401:725-740

43. Lee SC, Jeong HJ, Lee SK, Kim SJ. Hypoxic conditioned medium from human adipose-derived stem cells promotes mouse liver regeneration through JAK/STAT3 signaling. Stem Cells Transl Med 2016;5:816-825

44. Zou X, Gu D, Xing X, Cheng Z, Gong D, Zhang G, Zhu Y. Human mesenchymal stromal cell-derived extracellular vesicles alleviate renal ischemic reperfusion injury and enhance angiogenesis in rats. Am J Transl Res 2016;8:42894299

45. Zhang HC, Liu XB, Huang S, Bi XY, Wang HX, Xie LX, Wang YQ, Cao XF, Lv J, Xiao FJ, Yang Y, Guo ZK. Microvesicles derived from human umbilical cord mesenchymal stem cells stimulated by hypoxia promote angiogenesis both in vitro and in vivo. Stem Cells Dev 2012;21: 3289-3297 